\title{
Orbital dynamics during an ultrafast insulator to metal transition
}

\author{
Sergii Parchenko, ${ }^{1, *}, \dagger$ Eugenio Paris,,${ }^{1, *}$ Daniel McNally, ${ }^{1}$ Elsa Abreu, ${ }^{2}$ Markus Dantz, ${ }^{1}$ Elisabeth M. Bothschafter, ${ }^{1}$ \\ Alexander H. Reid $\odot,{ }^{3}$ William F. Schlotter, ${ }^{3}$ Ming-Fu Lin, ${ }^{3}$ Scott F. Wandel, ${ }^{3}$ Giacomo Coslovich $\odot{ }^{3}$, Sioan Zohar ${ }^{3},{ }^{3}$ \\ Georgi L. Dakovski, ${ }^{3}$ J. J. Turner, ${ }^{3,4}$ S. Moeller, ${ }^{3}$ Yi Tseng, ${ }^{1}$ Milan Radovic $\odot,{ }^{1}$ Conny Saathe, ${ }^{5}$ Marcus Agaaker $\odot,{ }^{5,6}$ \\ Joseph E. Nordgren ${ }^{\circ},{ }^{6}$ Steven L. Johnson, ${ }^{2,7}$ Thorsten Schmitt, ${ }^{1, \ddagger}$ and Urs Staub ${ }^{1, \S}$ \\ ${ }^{1}$ Swiss Light Source, Paul Scherrer Institute, CH-5232 Villigen PSI, Switzerland \\ ${ }^{2}$ Institute for Quantum Electronics, ETH Zürich, 8093 Zürich, Switzerland \\ ${ }^{3}$ LCLS, SLAC National Accelerator Laboratory, Menlo Park, California 94025, USA \\ ${ }^{4}$ Stanford Institute for Materials and Energy Sciences, SLAC National Accelerator Laboratory and Stanford University, 2575 Sand Hill Rd., \\ Menlo Park, California 94025, USA \\ ${ }^{5}$ Lund University, MAX IV Laboratory, Box 118, SE-22100 Lund, Sweden \\ ${ }^{6}$ Uppsala University, Department of Physics \& Astronomy, S-75120 Uppsala, Sweden \\ ${ }^{7}$ SwissFEL, Paul Scherrer Institut, CH-5232 Villigen PSI, Switzerland
}

(Received 19 November 2019; revised manuscript received 5 March 2020; accepted 1 April 2020; published 30 April 2020)

\begin{abstract}
We present ultrafast resonant inelastic x-ray scattering (RIXS) experiments performed at the vanadium $L$ edge to track changes in the electronic structure of $\mathrm{V}_{2} \mathrm{O}_{3}$, a classical Mott-Hubbard material. The probed orbital excitations within the $d$ shell of the $\mathrm{V}$ ion show a sub-ps time response, which evolves at later times to a state that appears electronically indistinguishable from the high-temperature metallic state. For low excitation fluences, a transient recovery or delay is observed, which could be related to a transient dimerization of the V-V bonds. Our results demonstrate the great potential for RIXS spectroscopy to study the ultrafast orbital dynamics in strongly correlated materials.
\end{abstract}

DOI: 10.1103/PhysRevResearch.2.023110

\section{INTRODUCTION}

As seen in many materials, a temperature-driven insulator to metal transition (IMT) is characterized by a sudden resistivity change upon heating that typically spans several orders of magnitude [1]. IMTs often involve a complex interplay of microscopic interactions including structural, electronic, and magnetic degrees of freedom. In many cases, IMTs and similar phase transitions can be triggered by an intense pulse of light [2-6]. These light-driven transitions often involve intermediate states that are not present in equilibrium and are visible only using time-resolved methods [7].

The IMT in $\mathrm{V}_{2} \mathrm{O}_{3}$ has been widely studied in equilibrium [8-10]. $\mathrm{V}_{2} \mathrm{O}_{3}$ undergoes a Mott-Hubbard IMT at $160 \mathrm{~K}$, transforming from an antiferromagnetic insulating phase to a paramagnetic metallic phase [10]. The IMT is accompanied

\footnotetext{
${ }^{*}$ These authors contributed equally to this work.

${ }^{\dagger}$ Present address: Laboratory for Mesoscopic Systems, Department of Materials, ETH Zurich, 8093 Zurich, Switzerland, Paul Scherrer Institut, 5232 Villigen PSI, Switzerland.

¥thorsten.schmitt@psi.ch

${ }^{\S}$ urs.staub@psi.ch
}

Published by the American Physical Society under the terms of the Creative Commons Attribution 4.0 International license. Further distribution of this work must maintain attribution to the author(s) and the published article's title, journal citation, and DOI. by a structural phase transition from a low-temperature (LT) monoclinic phase to a high-temperature (HT) rhombohedral corundum phase. The primary mechanism for the IMT is ascribed to electron correlation effects [11]. Although the formal oxidation state of the $\mathrm{V}$ ions in $\mathrm{V}_{2} \mathrm{O}_{3}$ is $\mathrm{V}^{3+}$ (valence shell configuration $\mathrm{V} 3 d^{2}$ ), the actual electronic configuration of the system, as well as the nature of the orbital structure, is still unclear. The orbital occupation has been investigated by polarization-dependent x-ray absorption spectroscopy [12], which indicated a high-spin state electron configuration $3 d^{2}$ for the $\mathrm{V}$ ions that have a different orbital occupancy in the different phases. More recently, a local density approximationdynamical mean-field theory study [13] predicts that the IMT is driven by correlation-induced enhancement of the crystalfield splitting.

The ultrafast dynamics of laser-excited $\mathrm{V}_{2} \mathrm{O}_{3}$ has been studied using a variety of methods [14-17]. Optical studies found strong changes in the speed of sound (acoustic phonons) through the IMT [15]. More recently, a long-lived transient electronic state lasting several picoseconds was revealed in the laser-excited HT phase of $\mathrm{V}_{2} \mathrm{O}_{3}$ by a combination of ultrafast angle-resolved photoemission (ARPES), x-ray diffraction, and optical reflectivity experiments [16]. This transient state was found to be associated with an increased dimerization of the $\mathrm{V}-\mathrm{V}$ ions occurring around $1 \mathrm{ps}$ after excitation. However, ARPES and optical reflectivity measurements of the timedependent electronic structure do not give direct information on the occupied and unoccupied orbitals involved in the ultrafast dynamics of laser-induced IMTs. A particularly powerful 
technique to study the elemental and orbital resolved electronic structure is resonant inelastic $\mathrm{x}$-ray scattering (RIXS). In RIXS the photon energy is tuned to an absorption edge of an atom to initiate an electronic transition between a core level and an unoccupied valence band state. The intermediate core-excited state relaxes by recombination of a valence electron with the core hole on a femtosecond timescale thereby re-emitting a photon. This leaves the material in an excited state that represents directly an elementary excitation of the charge, orbital, magnetic or lattice system [18-21]. RIXS has been previously employed to study $\mathrm{VO}_{2}$ [22] and $\mathrm{V}_{2} \mathrm{O}_{3}$, [23] although for the latter case it provided information only on the overall tendencies of the orbital physics due to limited energy resolution. Very few ultrafast RIXS studies on solids have been performed to date in the soft $[24,25]$ or hard $\mathrm{x}$-ray regimes [26].

Here we present a RIXS study of the ultrafast response of the $d$-d excitations in $\mathrm{V}_{2} \mathrm{O}_{3}$ after photoexcitation of the insulating state, with sufficient time and energy resolution to characterize the transient evolution of the $\mathrm{V} 3 d$ orbitals. We find an ultrafast sub-ps change in the $d$ - $d$ excitations between the different $\mathrm{V} 3 d-t_{2 g}$ orbitals followed by a transient recovery at intermediate (ps) timescale and a slower, quasithermal IMT.

\section{EXPERIMENTS}

High quality $\mathrm{V}_{2} \mathrm{O}_{3}$ thin films were grown with (001) outof-plane direction on (0001)-oriented $\mathrm{Al}_{2} \mathrm{O}_{3}$ substrates with pulsed laser deposition. The epitaxial growth conditions are achieved with an oxygen partial pressure of $3 \times 10^{-7} \mathrm{mbar}$ while the temperature of the substrate is kept at $750^{\circ} \mathrm{C}$. The $\mathrm{V}_{2} \mathrm{O}_{3}$ polycrystalline target was ablated using a $\mathrm{KrF}$ excimer laser $(\lambda=248 \mathrm{~nm})$ with $2 \mathrm{~Hz}$ repetition rate and a fluence of $\sim 1 \mathrm{~J} / \mathrm{cm}^{2}$. The film thickness obtained was $\sim 100 \mathrm{~nm}$. Further details on the sample characterization can be found in the Supplemental Material [27]. Preliminary characterization to determine the damage threshold of the sample was performed at normal incidence and revealed no visible damage of the film up to an incident optical laser fluence of $100 \mathrm{~mJ} / \mathrm{cm}^{2}$.

The static V L-edge RIXS measurements were carried out at the ADRESS beamline of the Swiss Light Source at the Paul Scherrer Institut, Switzerland [28]. The high-brilliance x-ray beam was monochromatized using a plane grating monochromator and focused down to a spot size of $\leqslant 4 \times 55 \mu \mathrm{m}^{2}$ at the sample position using an elliptical refocusing mirror. The incoming radiation was linearly polarized, with the polarization vector parallel to the scattering plane. The scattering angle was set to $2 \theta=90^{\circ}$, while all measurements were performed at the specular condition, i.e., with the incoming beam impinging at an angle of approximately $45^{\circ}$ with respect to the sample surface. The combined energy resolution was $55 \mathrm{meV}$ full width at half maximum (FWHM), determined by collecting the elastic scattering from a carbon tape reference.

The time-resolved experiments were performed at the Linac Coherent Light Source (LCLS) free electron laser using the resonant inelastic scattering spectrometer recently established at the soft $\mathrm{x}$-ray materials science (SXR) beamline [29]. Figure 1(b) shows the static $\mathrm{V} L_{2,3}$-edge $\mathrm{x}$-ray absorption spectrum collected in total fluorescence yield mode at SXR. The $\mathrm{x}$-ray beam arrives with an angle of $20^{\circ}$ with respect to (a)

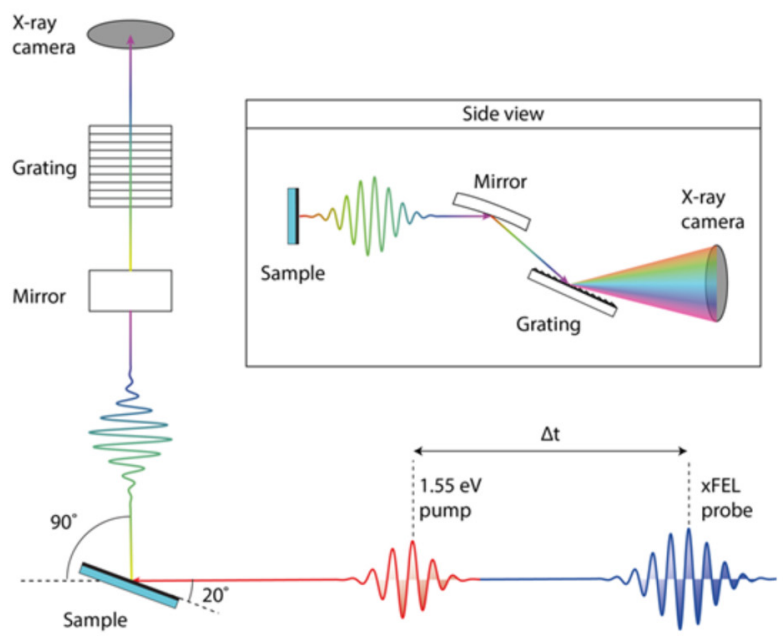

(b)

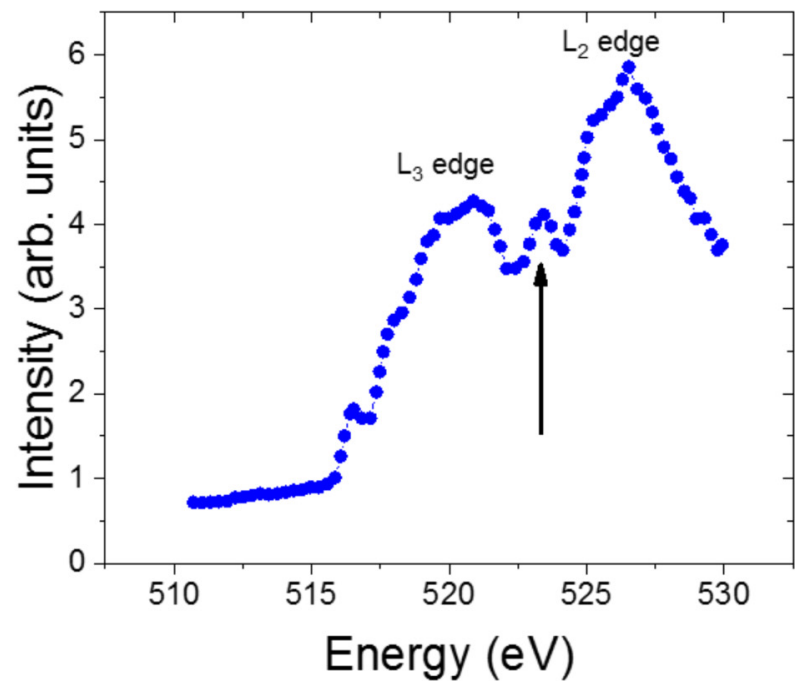

FIG. 1. (a) Schematic of the RIXS spectrometer setup at the SXR beamline of the LCLS. (b) $\mathrm{V} L_{2,3}$-edge x-ray absorption spectrum of $\mathrm{V}_{2} \mathrm{O}_{3}$ recorded in total fluorescence yield mode at the LCLS. The arrow points to the incident energy at the pre-edge of the $L_{2}$ resonance chosen for the time-resolved RIXS experiments.

the sample surface. The fluorescence signal is collected with a microchannel plate detector located at an angle of $90^{\circ}$ with respect to the incoming $x$-ray beam. The arrow indicates the incident $\mathrm{x}$-ray energy chosen, located at the pre-edge of the $L_{2}$ resonance, for the time-resolved RIXS experiment. This energy is optimal as it shows the highest intensity of the inelastic response and completely separates the fluorescence contribution from the $d-d$ excitations.

The experimental configuration for time-resolved RIXS is shown schematically in Fig. 1(a). We used 800 $\mathrm{nm} p$-polarized ultrashort laser pulses as a pump and $120 \mathrm{fs} \pi$-polarized $\mathrm{x}$-ray pulses as a probe. The $\pi$-polarized $\mathrm{x}$ rays reduce the elastic scattering probability due to the polarization dependence of Thomson scattering at the Brewster angle. Pump and probe beams propagate collinearly with $20^{\circ}$ incidence angle from the sample surface and were focused to $5370 \times 1330 \mu \mathrm{m}^{2}$ and $2050 \times 50 \mu \mathrm{m}^{2}$ spot sizes, 
respectively. The x-ray beam was monochromatized using a plane grating monochromator and focused on the sample using Kirkpatrick-Baez optics. The scattering angle was set to $90^{\circ}$. We estimate the combined energy resolution to be 200 meV FWHM, obtained by operating the beamline with $50-\mu \mathrm{m}$ exit slits and with the spectrometer grating set to the second diffraction order. The variable-line-space grating disperses the scattered $\mathrm{x}$ rays vertically allowing it to collect a larger solid angle in the horizontal plane; this allowed an extended horizontal line focus to be used that reduces sample damage by the x-rays without increasing the time to collect the signal. We used an Andor Newton DO940P charge coupled device (CCD) camera to collect the RIXS signal. Due to high readout noise and limited frame rate of the CCD camera we were not able to make a "shot-to-shot" data readout. Instead, we exposed the camera continuously for $10 \mathrm{~min}$ to obtain enough statistics for one RIXS spectrum. This approach makes a time jitter correction of the X-ray pulses for the arrival time impossible, which limits the time resolution. Figure S1(a) and S1(b) in the Supplemental Material (SM) show histograms of incoming $\mathrm{x}$-ray pulses and time jitter within $10 \mathrm{~min}$ of accumulation. A two-peak distribution of arrival time fluctuations is commonly observed at LCLS, resulting in a temporal uncertainty of the pump-probe delay of approximately $0.4 \mathrm{ps}$. Manual adjustments of the delay between pump and probe were performed when the short time average (10-s range) deviated more than 0.2 ps from the long term average.

To avoid artifacts due to the cosmic background radiation within the acquisition time, a threshold-based algorithm was implemented to filter the signal (see SM). The energy calibration was performed by recording the $L_{\alpha}$ and $L_{\beta}$ emission lines of a reference $\mathrm{Zn}$ foil, collected using the fourth order diffraction from the spectrometer grating.

\section{RESULTS}

Figure 2 shows a comparison of a V L-edge RIXS spectrum in the insulating LT state at a temperature of $20 \mathrm{~K}$ recorded at the ADRESS beamline of the Swiss Light Source with a spectrum recorded under similar conditions (except with significantly lower energy resolution) at a temperature of $50 \mathrm{~K}$ using the RIXS setup at the SXR beamline of the x-ray free electron laser LCLS at the SLAC National Accelerator Laboratory. The spectra show an intense peak at 0.2-eV energy loss, followed by several overlapping less intense peaks, located between 1 and $4 \mathrm{eV}$. The low energy peaks (A and B) possess a Raman-like behavior and are interpreted to be orbital $d$ - $d$ excitations. In this material, the trigonal crystal field splits the fivefold degenerate $3 d$ orbitals into a nondegenerate $a_{1 g}$ and two double-degenerate $e_{g}^{\pi}$ and $e_{g}^{\sigma}$ orbitals. Within the notation mentioned above, peak A is composed of low-energy $d$ - $d$ excitations involving orbitals of $a_{1 g}$ and $e_{g}^{\pi}$ symmetry, while peak B involves orbitals of $e_{g}^{\sigma}$ symmetry in the final state, which are located at higher energy. The fine structures exhibited by peak B reflect the different possible orbital occupations in the final state. In addition, each component might be further split into a spin conserving and a spin-flip channel as, e.g., observed in Refs. [30,31]. The region between 5 and $10 \mathrm{eV}$ energy loss is composed of charge transfer (CT)

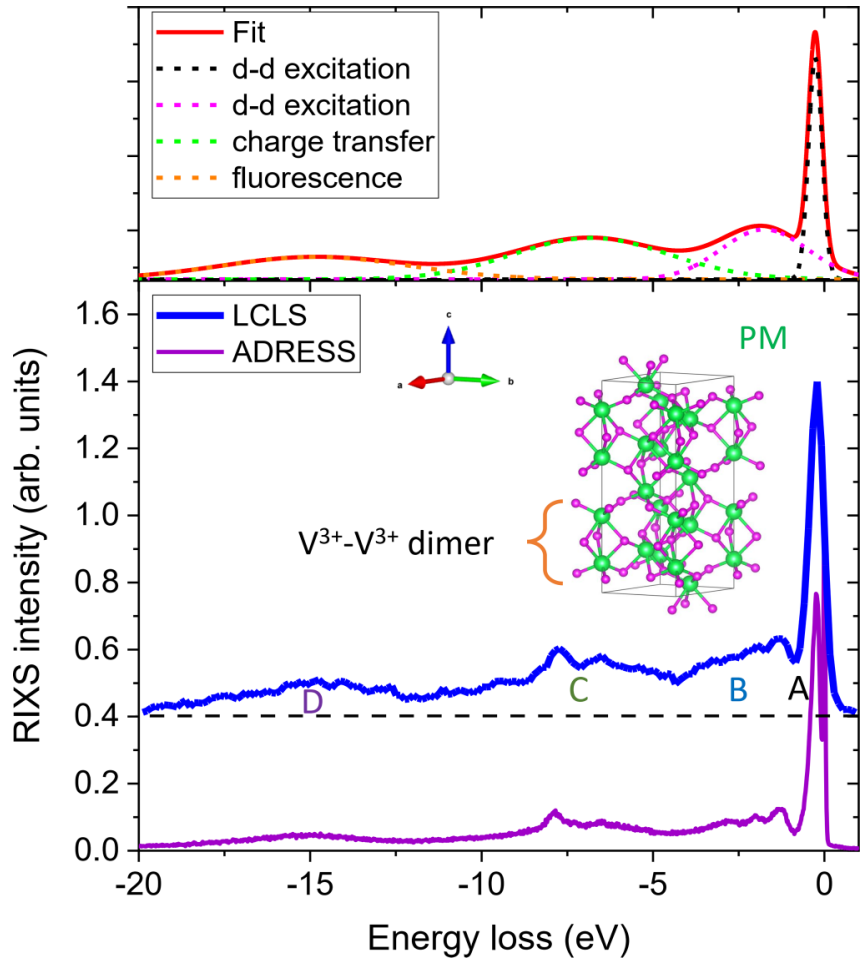

FIG. 2. RIXS spectra of insulating $\mathrm{V}_{2} \mathrm{O}_{3}$ recorded at the SXR beamline of the LCLS at $50 \mathrm{~K}$ (blue curve: middle) and at the ADRESS beamline of the Swiss Light Source at $20 \mathrm{~K}$ (violet curve: bottom). Both spectra are normalized to the main peak intensity at $\sim 0.2 \mathrm{eV}$. The spectra are vertically shifted for better visibility. Contribution to spectra from $d$ - $d$ excitations $(\mathrm{A}, \mathrm{B})$, charge transfer excitations (C), and fluorescence (D) are indicated. The inset shows the crystal structure for the high-temperature phase with indication of Vanadium dimers. The red line in the top panel is a fit to the sum of Gaussian functions for peak A, B, C, and D. The dashed curves show the individual components of the fit.

excitations (C), while above approximately 12-eV energy loss the spectrum contains mainly the non-Raman fluorescence response of the valence band, as found for vanadium oxides of different oxidation states (D) [32]. Peak $\mathrm{C}$ also contains spectral structures carrying information on the valence band and the metal-ligand hybridization. Both the $\mathrm{C}$ and $\mathrm{D}$ peaks have a marked fluorescencelike behavior, i.e., their energy depends linearly on the incoming energy. The spectra recorded at the LCLS are in good agreement with the spectra obtained at the synchrotron source, considering the difference in the energy resolution between the two data sets. The red line in the top panel of Fig. 2 represents a fit to a sum of four Gaussians (see $\mathrm{SM}$ ) representing the $\mathrm{A}, \mathrm{B}, \mathrm{C}$, and $\mathrm{D}$ contributions described above. We employ this simple model to better quantify the changes of the lowest-lying $d$ - $d$ excitation $(\sim 0.2 \mathrm{eV})$ in the RIXS spectra in our pump-probe experiment.

Figure 3(a) and 3(b) show proposed energy diagrams for the antiferromagnetic insulating (AFI) and paramagnetic metallic (PM) phases, respectively [33]. The 3d orbitals of $\mathrm{V}^{3+}$ contain two electrons, and in the crystal field this results in doubly occupied $e_{g}^{\pi}$ orbitals. Our static RIXS data indicate that the first unoccupied orbital $\left(a_{1 g}\right)$ is located at $\sim 0.2 \mathrm{eV}$ above the $e_{g}^{\pi}$ orbital, followed by $e_{g}^{\sigma}$ orbitals at significantly 


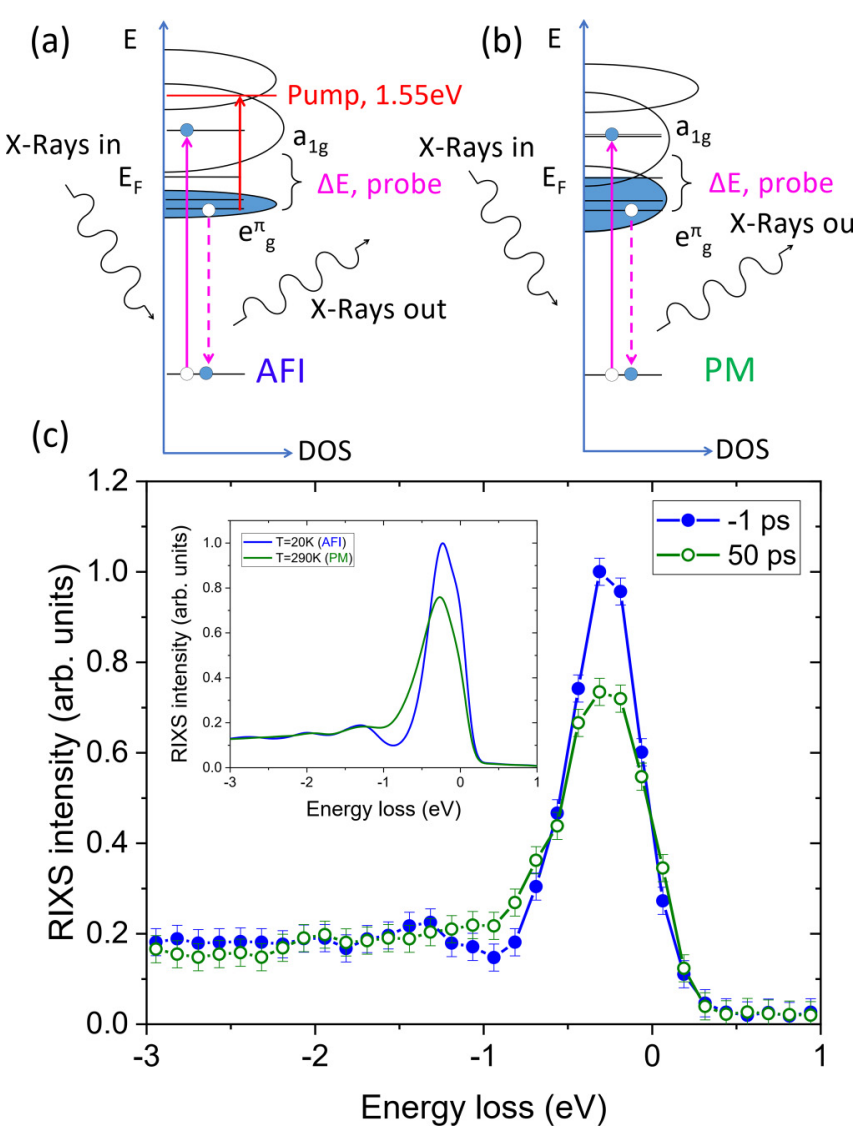

FIG. 3. Schematic energy diagram for (a) AFI and (b) PM phase $(50 \mathrm{~K})$, visualizing the pumping process and the subsequent RIXS excitations in the two phases. (c) Energy loss RIXS spectra covering the lowest lying $d$ - $d$ excitations $A$ before and after photoexcitation with a fluence of $\Phi=12 \mathrm{~mJ} / \mathrm{cm}^{2}$. The inset shows static RIXS data for AFI $(20 \mathrm{~K})$ and PM $(200 \mathrm{~K})$ phases that are convolved with the energy resolution of the time-resolved experiment.

higher energies [33]. In the AFI state, the crystal structure is monoclinic with more localized electronic states, as compared to the high-temperature rhombohedral phase (see Fig. 2 inset). This effect can also be seen in the $d$ - $d$ excitation, having a narrower $e_{g}^{\pi}$ to $a_{1 g}$ transition (A) at $20 \mathrm{~K}$ compared to room temperature as seen in Fig. 3(c) inset. A similar effect has recently been observed in the IMT of $\mathrm{NdNiO}_{3}$ [34]. These equilibrium spectra, measured at a synchrotron source, are convolved with the energy resolution of the time-resolved experiment to better identify possible spectral differences. A Gaussian fit to the $d$ - $d$ excitation A of the equilibrium data shows that the total spectral weight of this excitation remains constant across the phase transition within the experimental accuracy, although the width and peak height of A are strongly affected by the IMT. In the more delocalized PM state, the states are broader, representing an overlap of the $a_{1 g}$ band with the conduction band, making $\mathrm{V}_{2} \mathrm{O}_{3}$ metallic without a significant change in the energy level position. $\mathrm{VO}_{2}$ showed similar RIXS spectra when passing through its IMT, where the broadening was interpreted as originating from an additional magnetic interaction induced by singlet-triplet splitting [22].

Independent of the microscopic origin of the broadening, our time-resolved $\mathrm{V}_{2} \mathrm{O}_{3}$ data indicate that the laser excitation

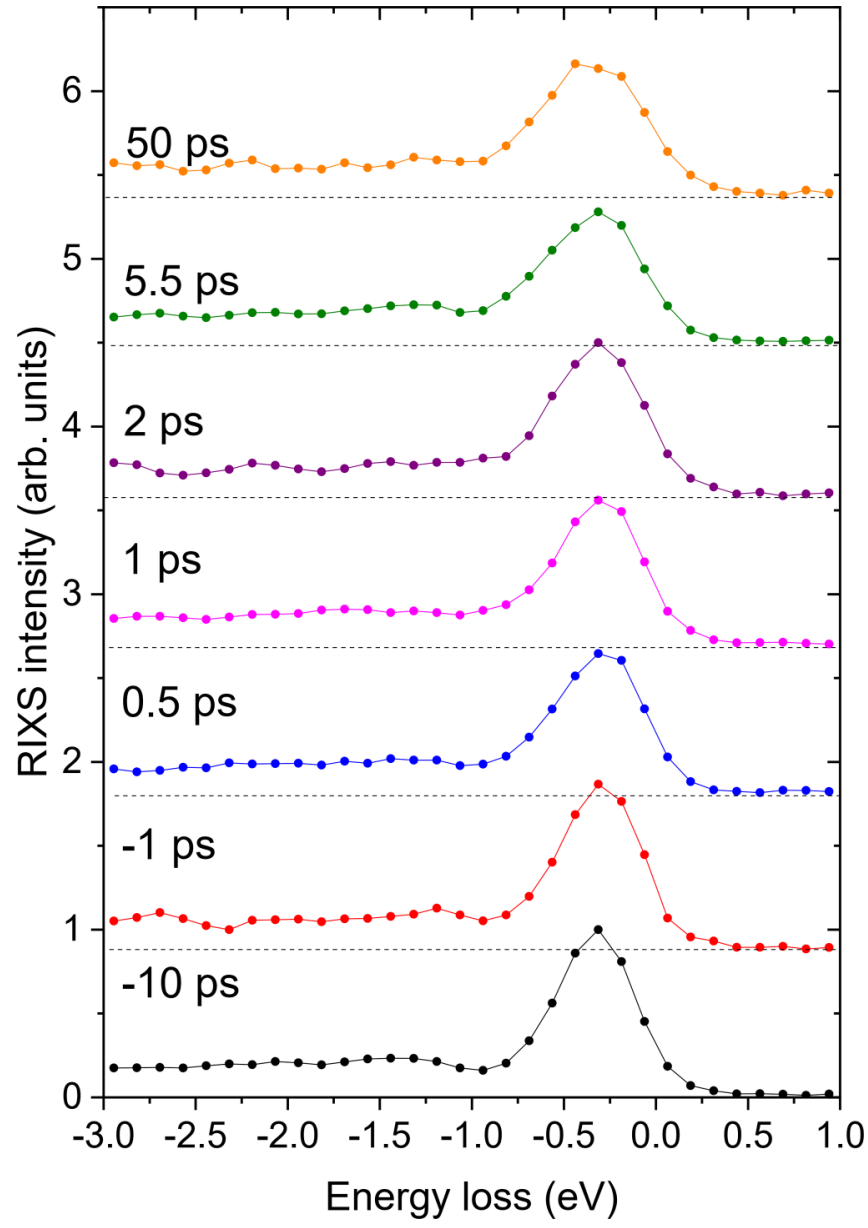

FIG. 4. $L$-edge RIXS spectra of $\mathrm{V}_{2} \mathrm{O}_{3}$ recorded at various time delays taken at $T=50 \mathrm{~K}$. The spectra are vertically offset for better clarity.

does induce the IMT. In fact, the static PM RIXS spectra closely resemble the spectra recorded $50 \mathrm{ps}$ after the excitation, as can be seen from comparing Fig. 3(c) with its inset. Note that the slightly different peak position between the transient data and the static data presented in the inset to panel 3 (c), is within the energy resolution of the time-resolved experiment. The change of the peak height of the low-energy $d$ - $d$ excitation (peak A) between the antiferromagnetic insulating and the paramagnetic metallic states in thermal equilibrium (using the static data convolved with the experimental resolution of the pump-probe setup) is similar to the change observed in the dynamic data; $R_{\text {equilibrium }}=\frac{I_{20 \mathrm{~K}}}{I_{200 \mathrm{~K}}}=1.31 \pm 0.02$ compared to the dynamic ratio $R_{\text {dynamic }}=\frac{I_{-1 \mathrm{ps}}}{I_{50 \mathrm{ps}}}=1.29 \pm$ 0.06 . We note that in the static RIXS experiment, the center of gravity of peak A shifts to higher loss energies from LT phase to the HT phase [see the inset of Fig. 3(c)]. Such an effect is not observed in our time-resolved experiment, mainly due to the limited energy resolution and statistics available.

Figure 4 shows the $\mathrm{V}$ L-edge RIXS spectra of $\mathrm{V}_{2} \mathrm{O}_{3}$ collected at various delay times at a temperature of $50 \mathrm{~K}$ and a laser fluence of $12 \mathrm{~mJ} / \mathrm{cm}^{2}$. The data collection was performed in acquisition runs of $10 \mathrm{~min}$ integration and normalized by the time integrated $I_{0}$, while several runs were 

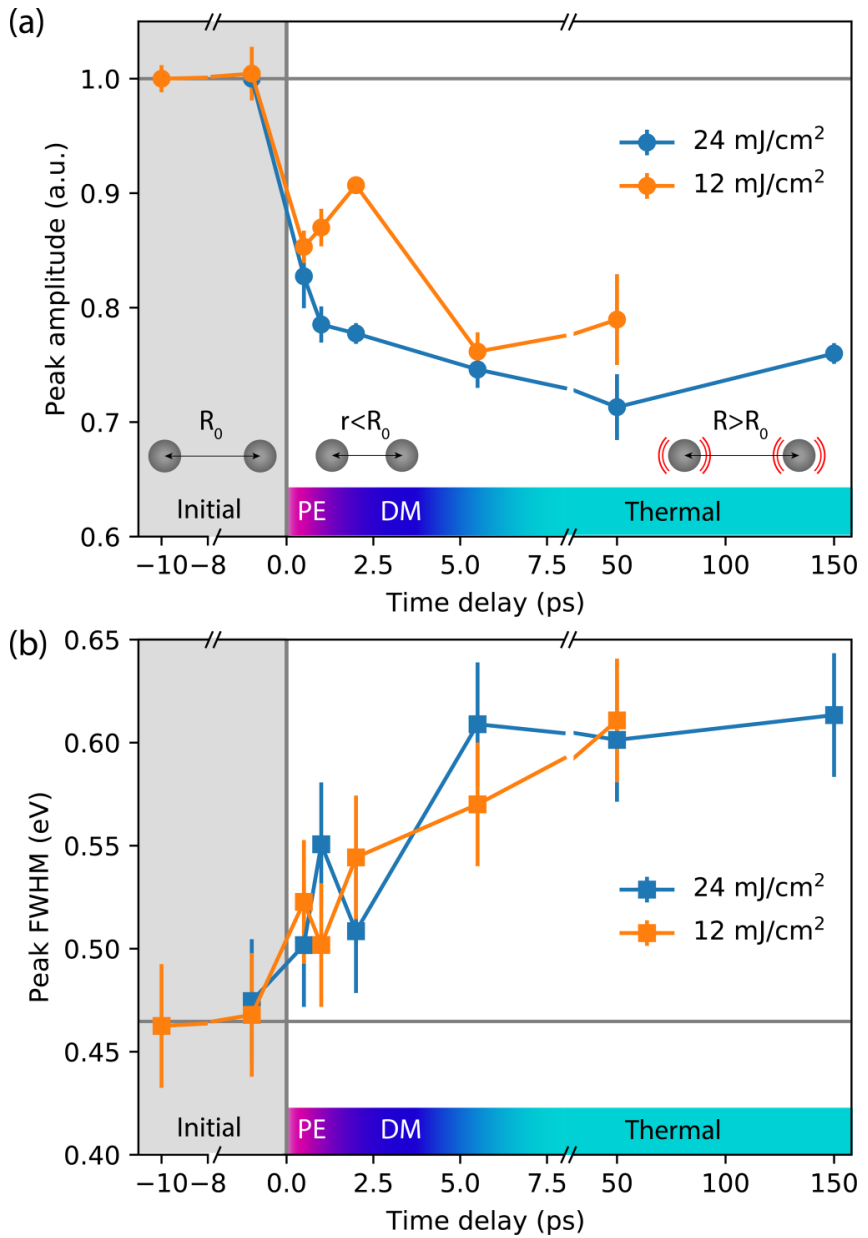

FIG. 5. Time dependence of the RIXS peak amplitude (a) and the FWHM (b) for two pump laser fluences. The color bar on the bottom of panel (a) refers to different transient states: the initial state; the immediate photoexcited state (PE); the "dimerized" state (DM) and the thermalizing state (Thermal).

acquired for each given time delay. To extract details on the transient changes of the RIXS spectra we fitted the d-d, charge-transfer excitations and the fluorescence excitations with a total of 4 Gaussian functions, as shown in Fig. 2. No significant changes are visible at different time delays for the higher lying $d-d$ excitations $\mathrm{B}$, centered around $2.5 \mathrm{eV}$, for the CT excitations $\mathrm{C}$, centered around $7.5 \mathrm{eV}$, or for the fluorescence contribution D, centered around $15 \mathrm{eV}$. We keep all parameters used to fit these excitations to the values obtained for the data taken before $t_{0}$, refining only the parameters related to the $e_{g}^{\pi}-a_{1 g} d-d$ excitation, located at $<1 \mathrm{eV}$ energy loss.

The time scale of this laser-induced transition can be explored using intermediate pump-probe delay times. Figure 5 shows the time evolution of the peak height (a) and width (b) of the $0.2-\mathrm{eV} d-d$ excitation normalized to value(s) before time zero, as obtained from fitting the RIXS data collected with 12 and $24 \mathrm{~mJ} / \mathrm{cm}^{2}$ incident laser pump fluences. Both the peak width and height change drastically within the first $5 \mathrm{ps}$ from the pump pulse. On shorter time scales, we see that the peak height for both fluences drops significantly on a time scale of less than one picosecond [photoexcited state (PE)]. Optical pump-probe experiments show changes in the optical conductivity over several tens of femtoseconds [16], suggesting that these changes in the RIXS signal could be much faster than we are able to observe here. This initial change is likely due to the laser-driven redistribution of electrons, resulting in a depopulation of the vanadium $3 \mathrm{~d}$ valence band as electrons are promoted into the conduction band.

\section{DISCUSSION}

The dynamics measured for the two fluences differ on intermediate time scales, between 1 and 5 ps. After 1 ps the amplitude of the $e_{g}^{\pi}-a_{1 g} d-d$ peak decreases further for the $24 \mathrm{~mJ} / \mathrm{cm}^{2}$ data. In contrast, the data for $12 \mathrm{~mJ} / \mathrm{cm}^{2}$ fluence appears to plateau or slightly recover over a 2-ps timescale. The time scale of this possible partial recovery is similar to that of the transient dimerization (DM) of $\mathrm{V}-\mathrm{V}$ pairs observed in the PM phase by ultrafast $\mathrm{x}$-ray diffraction [16]. The reduced V-V pair distance observed has been interpreted as a direct consequence of the electronic excitation of the system $[14,16]$. Since the V-V dimerization is not strongly influenced by the phase transition, it is plausible to speculate that a similar effect might also occur when the system is photoexcited in the low-temperature phase. The smaller interionic V-V distances induced by the dimerization would increase the Coulomb repulsion, which in turn leads to an increase in localization of the $\mathrm{V} 3 d$ electrons, as in the case for the thermally driven IMT in $\mathrm{VO}_{2}[1,35]$. This localization partially counteracts the delocalization caused by the metal-insulator transition, resulting in an apparent delay of the drop of the $d-d$ peak. As the system relaxes to local thermal equilibrium, the highly excited electrons transfer heat to the lattice and the electronically induced V-V dimerization relaxes to its value in thermal equilibrium at an elevated temperature within the PM phase.

\section{CONCLUSION}

In summary, we demonstrated that femtosecond timeresolved RIXS with energy and time resolution allows us to see intermediate nonthermal states existing during the photoexcited ultrafast IMT in $\mathrm{V}_{2} \mathrm{O}_{3}$. By tracking the time-delay dependence of the $d-d$ excitations, we find indication of a possible delay of the electronic signatures of the phase transition when the system is driven at low fluences $\left(12 \mathrm{~mJ} / \mathrm{cm}^{2}\right)$. We propose that this phenomenon can be explained with a transient dimerization of vanadium ions, induced by the pump pulse. Combined ultrafast diffraction and RIXS measurements of the laser-induced IMT would provide a useful confirmation of this scenario. Together with the recent work pointing out the importance of disorder through an IMT in $\mathrm{VO}_{2}$ [36], these results show that additional microscopic information on the dynamics of the electronic states is required to understand IMT's in strongly correlated electron systems.

\section{ACKNOWLEDGMENTS}

E.A. acknowledges support from the ETH Zurich Postdoctoral Fellowship Program and from the Marie Curie 
Actions for People COFUND Program. E.M.B. acknowledges funding from the European Community's Seventh Framework Programme (FP7/2007-2013) under Grant No. 290605 (PSIFELLOW/COFUND). Financial support from the Swiss National Science Foundation and its National Center of Competence in Research, Molecular Ultrafast Science and Technology (NCCR MUST) is greatly acknowledged. The static synchrotron experiments have been performed at the ADRESS beamline of the Swiss Light Source at the Paul Scherrer Institut (PSI). D.M. was supported by the Swiss National Science Foundation through the NCCR MARVEL. E.P. and Y.T. were supported by the Sinergia network Mott Physics Beyond the
Heisenberg Model (MPBH) (SNSF Research Grants No. CRSII2_160765/1 and No. CRSII2_141962). M.D. was partially funded by the Swiss National Science Foundation within the D-A-CH programme (SNSF Research Grant No. 200021L 141325). J.J.T. acknowledges support from the U.S. Department of Energy, Office of Science, Basic Energy Sciences through the Early Career Research Program. This work used the Linac Coherent Light Source (LCLS), SLAC National Accelerator Laboratory, and was supported by the U.S. Department of Energy, Office of Science, Office of Basic Energy Sciences, Material Sciences and Engineering Division, under Contract No. DE-AC02-76SF00515.
[1] M. Imada, A. Fujimori, and Y. Tokura, Rev. Mod. Phys. 70, 1039 (1998).

[2] A. Cavalleri, C. Toth, C. W. Siders, J. A. Squier, F. Raksi, P. Forget, and J. C. Kieffer, Phys. Rev. Lett. 87, 237401 (2001).

[3] D. Polli, M. Rini, S. Wall, R. W. Schoenlein, Y. Tomioka, Y. Tokura, G. Cerullo, and A. Cavalleri, Nat. Mater. 6, 643 (2007).

[4] F. Schmitt, P. S. Kirchmann, U. Bovensiepen, R. G. Moore, L. Rettig, M. Krenz, J.-H. Chu, N. Ru, L. Perfetti, D. H. Lu, M. Wolf, I. R. Fisher, and Z.-X. Shen, Science 321, 1649 (2008).

[5] R. Yusupov, T. Mertelj, V. V. Kabanov, S. Brazovskii, P. Kusar, J. H. Chu, I. R. Fisher, and D. Mihailovic, Nat. Phys. 6, 681 (2010).

[6] M. Eichberger, H. Schafer, M. Krumova, M. Beyer, J. Demsar, H. Berger, G. Moriena, G. Sciaini, and R. J. D. Miller, Nature (London) 468, 799 (2010).

[7] P. Beaud, A. Caviezel, S. O. Mariager, L. Rettig, G. Ingold, C. Dornes, S.-W. Huang, J. A. Johnson, M. Radovic, T. Huber, T. Kubacka, A. Ferrer, H. T. Lemke, M. Chollet, D. Zhu, J. M. Glownia, M. Sikorski, A. Robert, H. Wadati, M. Nakamur, M. Kawasaki, Y. Tokura, S. L. Johnson, and U. Staub, Nat. Mater. 13, 923 (2014).

[8] D. B. McWhan and J. P. Remeika, Phys. Rev. B 2, 3734 (1970).

[9] D. B. McWhan, A. Menth, J. P. Remeika, W. F. Brinkman, and T. M. Rice, Phys. Rev. B 7, 1920 (1973).

[10] R. M. Moon, Phys. Rev. Lett. 25, 527 (1970).

[11] G. A. Thomas, D. H. Rapkine, S. A. Carter, A. J. Millis, T. F. Rosenbaum, P. Metcalf, and J. M. Honig, Phys. Rev. Lett. 73, 1529 (1994).

[12] J. H. Park, L. H. Tjeng, A. Tanaka, J. W. Allen, C. T. Chen, P. Metcalf, J. M. Honig, F. M. F. de Groot, and G. A. Sawatzky, Phys. Rev. B 61, 11506 (2000).

[13] A. I. Poteryaev, J. M. Tomczak, S. Biermann, A. Georges, A. I. Lichtenstein, A. N. Rubtsov, T. Saha-Dasgupta, and O. K. Andersen, Phys. Rev. B 76, 085127 (2007).

[14] A. Singer, J. G. Ramirez, I. Valmianski, D. Cela, N. Hua, R. Kukreja, J. Wingert, O. Kovalchuk, J. M. Glownia, M. Sikorski, M. Chollet, M. Holt, I. K. Schuller, and O. G. Shpyrko, Phys. Rev. Lett. 120, 207601 (2018).

[15] E. Abreu, S. M. Wang, J. G. Ramirez, M. K. Liu, J. D. Zhang, K. Geng, I. K. Schuller, and R. D. Averitt, Phys. Rev. B 92, 085130 (2015).

[16] G. Lantz, B. Mansart, D. Grieger, D. Boschetto, N. Nilforoushan, E. Papalazarou, N. Moisan, L. Perfetti, V. L. R. Jacques, D. Le Bolloc'h, C. Laulhe, S. Ravy, J. P. Rueff, T. E.
Glover, M. P. Hertlein, Z. Hussain, S. Song, M. Chollet, M. Fabrizio, and M. Marsi, Nat. Commun. 8, 13917 (2017).

[17] F. Giorgianni, J. Sakai, and S. Lupi, Nat. Commun. 10, 1159 (2019).

[18] A. Kotani and S. Shin, Rev. Mod. Phys. 73, 203 (2001).

[19] L. J. P. Ament, M. van Veenendaal, T. P. Devereaux, J. P. Hill, and J. van den Brink, Rev. Mod. Phys. 83, 705 (2011).

[20] J. Schlappa, K. Wohlfeld, K. J. Zhou, M. Mourigal, M. W. Haverkort, V. N. Strocov, L. Hozoi, C. Monney, S. Nishimoto, S. Singh, A. Revcolevschi, J. S. Caux, L. Patthey, H. M. Ronnow, J. van den Brink, and T. Schmitt, Nature (London) 485, 82 (2012).

[21] L. Braicovich, J. van den Brink, V. Bisogni, M. M. Sala, L. J. P. Ament, N. B. Brookes, G. M. De Luca, M. Salluzzo, T. Schmitt, V. N. Strocov, and G. Ghiringhelli, Phys. Rev. Lett. 104, 077002 (2010).

[22] H. He, A. X. Gray, P. Granitzka, J. W. Jeong, N. P. Aetukuri, R. Kukreja, L. Miao, S. A. Breitweiser, J. Wu, Y. B. Huang, P. Olalde-Velasco, J. Pelliciari, W. F. Schlotter, E. Arenholz, T. Schmitt, M. G. Samant, S. S. P. Parkin, H. A. Durr, and L. A. Wray, Phys. Rev. B 94, 161119(R) (2016).

[23] C. F. Hague, J. M. Mariot, V. Ilakovac, R. Delaunay, M. Marsi, M. Sacchi, J. P. Rueff, and W. Felsch, Phys. Rev. B 77, 045132 (2008).

[24] M. Beye, F. Sorgenfrei, W. F. Schlotter, W. Wurth, and A. Fohlisch, Proc. Natl. Acad. Sci. USA 107, 16772 (2010).

[25] A. S. M. Ismail, Y. Uemura, S. H. Park, S. Kwon, M. Kim, H. Elnaggar, F. Frati, Y. Niwa, H. Wadati, Y. Hirata, Y. Zhang, K. Yamagami, S. Yamamoto, I. Matsuda, U. Halisdemir, G. Koster, B. M. Weckhuysen, and F. M. F. de Groot, Phys. Chem. Chem. Phys. 22, 2685 (2020).

[26] M. P. M. Dean, Y. Cao, X. Liu, S. Wall, D. Zhu, R. Mankowsky, V. Thampy, X. M. Chen, J. G. Vale, D. Casa, J. Kim, A. H. Said, P. Juhas, R. Alonso-Mori, J. M. Glownia, A. Robert, J. Robinson, M. Sikorski, S. Song, M. Kozina, H. Lemke, L. Patthey, S. Owada, T. Katayama, M. Yabashi, Y. Tanaka, T. Togashi, J. Liu, C. R. Serrao, B. J. Kim, L. Huber, C. L. Chang, D. F. McMorrow, M. Forst, and J. P. Hill, Nature Mater. 15, 601 (2016).

[27] See Supplemental Material at http://link.aps.org/supplemental/ 10.1103/PhysRevResearch.2.023110 for details on data collection and sample characterization.

[28] V. N. Strocov, T. Schmitt, U. Flechsig, T. Schmidt, A. Imhof, Q. Chen, J. Raabe, R. Betemps, D. Zimoch, J. Krempasky, X. 
Wang, M. Grioni, A. Piazzalunga, and L. Patthey, J. Synch. Radiat. 17, 631 (2010).

[29] W. F. Schlotter, J. J. Turner, M. Rowen, P. Heimann, M. Holmes, O. Krupin, M. Messerschmidt, S. Moeller, J. Krzywinski, R. Soufli, M. Fernandez-Perea, N. Kelez, S. Lee, R. Coffee, G. Hays, M. Beye, N. Gerken, F. Sorgenfrei, S. HauRiege, L. Juha, J. Chalupsky, V. Hajkova, A. P. Mancuso, A. Singer, O. Yefanov, I. A. Vartanyants, G. Cadenazzi, B. Abbey, K. A. Nugent, H. Sinn, J. Luning, S. Schaffert, S. Eisebitt, W. S. Lee, A. Scherz, A. R. Nilsson, and W. Wurth, Rev. Sci. Instrum. 83, 043107, (2012).

[30] E. Benckiser, L. Fels, G. Ghiringhelli, M. Moretti Sala, T. Schmitt, J. Schlappa, V. N. Strocov, N. Mufti, G. R. Blake, A. A. Nugroho, T. T. M. Palstra, M. W. Haverkort, K. Wohlfeld, and M. Grueninger, Phys. Rev. B 88, 205115 (2013).

[31] D. E. McNally, X. Y. Lu, J. Pelliciari, S. Beck, M. Dantz, M. Naamneh, T. Shang, M. Medarde, C. W. Schneider, V. N. Strocov, E. V. Pomjakushina, C. Ederer, M. Radovic, and T. Schmitt, Npj Quant. Mater. 4, 6 (2019).
[32] T. Schmitt, L. C. Duda, A. Augustsson, J. H. Guo, J. Nordgren, J. E. Downes, C. McGuinness, K. E. Smith, G. Dhalenne, A. Revcolevshci, M. Klemm, and S. Horn, Surf. Rev. Lett. 9, 1369 (2002).

[33] P. Hansmann, A. Toschi, G. Sangiovanni, T. Saha-Dasgupta, S Lupi, M. Marsi, and K. Held, Phys. Status Solidi B 250, 1251 (2013).

[34] V. Bisogni, S. Catalano, R. J. Green, M. Gibert, R. Scherwitzl, Y. B. Huang, V. N. Strocov, P. Zubko, S. Balandeh, J. M. Triscone, G. Sawatzky, and T. Schmitt, Nature Commun. 7, 13017 (2016).

[35] M. Abbate, F. M. F. de Groot, J. C. Fuggle, Y. J. Ma, C. T. Chen, F. Sette, A. Fujimori, Y. Ueda, and K. Kosuge, Phys. Rev. B 43, 7263 (1991).

[36] S. Wall, S. Yang, L. Vidas, M. Chollet, J. M. Glownia, M. Kozina, T. Katayama, T. Henighan, M. Jiang, T. A. Miller, D. A. Reis, L. A. Boatner, O. Delaire, and M. Trigo, Science 362, 572 (2018). 